\title{
Virtual reality simulation for surgery: from video games to transanal total mesorectal excision
}

\author{
B. Martin-Perez ${ }^{1} \cdot$ H. Bennis ${ }^{1} \cdot$ A. M. Lacy ${ }^{1}$
}

Received: 1 December 2017 / Accepted: 3 December 2017 / Published online: 18 December 2017

๑) Springer International Publishing AG, part of Springer Nature 2017

In order for surgeons to develop manual skills, training is fundamental. Hands-on training could be considered as the ideal form of training as it involves a direct and active participation of the trainee in the surgery. However, taking part in the surgery is frequently impossible or insufficient. Consequently, different training methods have been used in the past to enhance surgical training, from simple benchtop simulators, to elaborate video trainers [1, 2]. Straightforward procedures could be practiced on synthetic material or tissue from animal cadavers on the benchtop simulators. Box trainers were devised as plain boxes using a camera, screen, light source and instruments for laparoscopic training and evolved into video trainers, with motion sensors and movement economy trackers included.

Prior to the birth of virtual reality (VR), these training methods were the best tools trainees could count on to improve their manual skills. However, with the advent of new technologies, surgical training is slowly heading toward the virtual world, initially limited to video games but expanding to include educational tools. VR, occasionally referred to as immersive VR, is a "near-reality" artificial environment created with software that provides an immediate first-person experience in a simulation or recreation of reality. The main distinguishing characteristic of VR, unlike conventional three-dimensional (3D) or 360-degree images, is the control over the image and the interaction between the user and the environment, as the user has the capacity to decide where to look and how to navigate. New VR headsets, mainly designed for video game users, incorporate sensors to track head and eye movements, immersing the user in a given scenario. In contrast to VR, augmented reality (AR)

B. Martin-Perez

beatriz_martin1@yahoo.com

1 Department of General Surgery, Hospital Clinic of Barcelona, ICMDiM, Carrer de Villarroel, 170, 08036 Barcelona, Spain only delivers virtual elements to enhance and enrich reality but does not allow interaction with the environment.

When comparing VR to the traditional training methods, a meta-analysis of 30 studies demonstrated that VR simulation is as good as box trainers and significantly more effective than video trainers [1]. Hence, multiple medical specialties have already started to incorporate these innovations into their daily practice, mostly in surgery, resulting in many positive outcomes for surgical planning and simulation. In vascular surgery, VR makes it possible to analyze the spatial relationship between aneurysms and skull, when compared to plain 3D-images [3]. Patient-specific virtual reality rehearsal (PsR) before endovascular infrarenal aneurysm repair (EVAR) has been shown to reduce perioperative errors $(26 \%$ reduction of minor errors and $76 \%$ reduction of major errors) and to decrease the number of angiograms required to deploy the stent graft, thereby reducing delays. In neurosurgery, tractography is a specific VR technique that reconstructs neural tracts using data collected by diffusion tensor imaging (DTI), allowing simulation of neurosurgical interventions preoperatively $[4,5]$.

The field of general surgery is lagging behind other surgical specialties with regard to VR. Up to now, only pilot studies have been published about the use of VR in general surgery. These studies have shown that VR training tools improve the laparoscopic skills of both inexperienced and experienced surgeons [1]. The experience gained from other procedures indicates unequivocally that gastrointestinal surgery can benefit from VR training both in the preoperative and the intraoperative setting, especially in technically challenging procedures such as transanal total mesorectal excision (taTME) [6-8]. VR combined with 3D virtual patient-specific models will help in planning and simulating procedures in advance, locating essential structures such as ureters, vessels and neural bundles, which are key landmarks subjected to injury during taTME. Once in the operating room, VR images can guide surgical navigation, enhancing the surgeons' view of the operating field by combining the 
visual field with the radiology images. Fluorescence guidance by the augmented eye [9] is a non-invasive method that allows real-time cartography of structures (ureters and nerves), labeled by injected fluorophores, which are excited by near-infrared laser cameras [10]. Another asset of the VR glasses being developed is the ability to provide real-time microscopic images with confocal laser endomicroscopy (CLE) [9], which generates digital "biopsies" at micronlevel resolution. This technique could translate into a precise estimation of tumor margins and could end unnecessary sacrificing of the anal sphincters or reinterventions due to positive margins in oncologic resections. The augmented hand [9] is a flexible device that overcomes the rigidity of the laparoscopic instruments that could be the solution for the pure natural orifice transluminal endoscopic surgery (NOTES) taTME [11], where the dissection is performed completely transanally. With current rigid instruments, it is difficult to reach the splenic flexure, due to the angle formed by the promontory, while this VR minimally invasive "hand" enables surgical triangulation, overcoming this limitation.

Some hurdles need to be overcome in order for VR to become a useful daily tool for surgeons. One of the major drawbacks limiting the proliferation of the enhanced reality devices is the lack of haptic feedback on the VR controllers, a key capability that controllers are required to have. VR with haptic feedback is at least as effective in training as traditional box trainers and makes possible shorter operative times and more efficient movements [9]. Creating effective haptic sensors on the VR systems providing force and tactile feedback to the surgeon from the operative field via the laparoscopic instruments is challenging and still under development and evaluation, but will be essential for efficient training and simulation.

The advent of VR simulators has ushered in a new era, but these advances have not yet been fully translated into the clinical setting. Proficiency-based training, with optimal provision of immediate feedback and on the spot instructions, the motivation for deliberate practice and the availability of practicing varying levels of difficulty all seem to enhance the learning outcomes of VR simulation [1, 2, 5]. Different settings should be proposed for the surgeon, from simple laparoscopic cholecystectomy to more demanding procedures such as gastrectomies or total mesorectal excision. Performing a PsR before real taTME surgery based on VR images to foresee possible difficulties and complications, or navigating through the sensitive structures, should become part of daily practice on the near future, in order to improve patient safety while enhancing surgical precision.

\section{Compliance with ethical standards}

Conflict of interest The authors declare that they have no conflict of interest.

Ethical approval This article does not contain any studies with human participants or animals performed by any of the authors.

Informed consent For this type of study formal consent is not required.

\section{References}

1. Alaker M, Wynn GR, Arulampalam T (2016) Virtual reality training in laparoscopic surgery: a systematic review \& meta-analysis. Int J Surg 29:85-94. https://doi.org/10.1016/j.ijsu.2016.03.034

2. Hiemstra E, Terveer EM, Chmarra MK, Dankelman J, Jansen FW (2011) Virtual reality in laparoscopic skills training: is haptic feedback replaceable? Minim Invasive Ther Allied Technol 20:179-184. https://doi.org/10.3109/13645706.2010.532502

3. Desender LM, Van Herzeele I, Lachat ML, PAVLOV Study Group et al (2016) Patient-specific rehearsal before EVAR: influence on technical and nontechnical operative performance. A randomized controlled trial. Ann Surg 264(5):703-709

4. Ortiz-Catalan M, Sander N, Kristoffersen MB, Håkansson B, Brånemark R (2014) Treatment of phantom limb pain (PLP) based on augmented reality and gaming controlled by myoelectric pattern recognition: a case study of a chronic PLP patient. Front Neurosci 25(8):24. https://doi.org/10.3389/fnins.2014.00024

5. Akar JG, Hummel JP (2016) Editorial commentary: virtual medicine-a better reality? Trends Cardiovasc Med 26(8):731-732. https://doi.org/10.1016/j.tcm.2016.06.012

6. Lacy AM, Tasende MM, Delgado S et al (2015) Transanal total mesorectal excision for rectal cancer: outcomes after 140 patients. J Am Coll Surg 221:415-423. https://doi.org/10.1016/j. jamcollsurg.2015.03.046

7. Penna M, Hompes R, Arnold S, TaTME Registry Collaborative et al (2017) Transanal total mesorectal excision: international registry results of the first 720 cases. Ann Surg 266(1):111-117. https://doi.org/10.1097/SLA.0000000000001948

8. Burke JP, Martin-Perez B, Khan A et al (2016) Transanal total mesorectal excision for rectal cancer: early outcomes in 50 consecutive patients. Colorectal Dis 18:570-577. https://doi. org/10.1111/codi.13263

9. Franchini Melani AG, Diana M, Marescaux J (2016) The quest for precision in transanal total mesorectal excision. Tech Coloproctol 20(1):11-18. https://doi.org/10.1007/s10151-015-1405-3

10. Atallah S, Martin-Perez B, Drake J, Stotland P, Ashamalla S, Albert M (2015) The use of a lighted stent as a method for identifying the urethra in male patients undergoing transanal total mesorectal excision: a video demonstration. Tech Coloproctol 19:375. https://doi.org/10.1007/s10151-015-1297-2

11. Marks JH, Lopez-Acevedo N, Krishnan B, Johnson MN, Montenegro GA, Marks GJ (2016) True NOTES TME resection with splenic flexure release, high ligation of IMA, and side-to-end hand-sewn coloanal anastomosis. Surg Endosc 30:4626-4631. https://doi.org/10.1007/s00464-015-4731-7 